\title{
Unraveling the Role of microRNA and isomiRNA Networks in Multiple Primary Melanoma Pathogenesis
}

\section{Emi Dika}

University of Bologna

\section{Elisabetta Broseghini}

University of Bologna

Elisa Porcellini

University of Bologna

Martina Lambertini

University of Bologna

Mattia Riefolo

University of Bologna

Giorgio Durante

University of Bologna

Phillipe Loher

Thomas Jefferson University

\section{Roberta Roncarati}

Istituto di Ricerca Genetica e Biomedica Consiglio Nazionale delle Ricerche

\section{Cristian Bassi}

Universita degli Studi di Ferrara

\section{Cosimo Misciali}

Azienda Ospedaliero-Universitaria di Bologna Policlinico Sant'Orsola-Malpighi

\section{Massimo Negrini}

Universita degli Studi di Ferrara

\section{Isidore Rigoutsos}

Thomas Jefferson University

\section{Eric Londin}

Thomas Jefferson University

\section{Annalisa Patrizi}

Universita di Bologna

Manuela Ferracin ( $\nabla$ manuela.ferracin@unibo.it)

Universita degli Studi di Bologna https://orcid.org/0000-0002-1595-6887 
Research

Keywords: microRNA, isomiRNA, Malignant cutaneous melanoma (CM), epithelial-mesenchymal transition (EMT)

Posted Date: July 21st, 2020

DOI: https://doi.org/10.21203/rs.3.rs-44044/v1

License: (c) (i) This work is licensed under a Creative Commons Attribution 4.0 International License.

Read Full License 


\section{Abstract}

Background Malignant cutaneous melanoma (CM) is a potentially lethal form of skin cancer whose worldwide incidence has been constantly increasing over the past decades. During their lifetime about $8 \%$ of patients with CM will develop multiple primary melanomas (MPM). Patients affected by MPM could have a genetically determined susceptibility, though germline mutations in hereditary melanoma genes are rarely detected.

Methods To better characterize the biology of this subset of melanomas, we explored the miRNome of 24 single and multiple primary melanomas, including multiple tumors from the same patient, using a smallRNA sequencing approach and bioinformatic detection of miRNA isoforms. The differential expression of specific miRNAs/isomiRs was obtained using quantitative PCR.

Results From a supervised analysis, 22 miRNAs were differentially expressed in MPM compared to single $\mathrm{CM}$, including key miRNAs involved in epithelial-mesenchymal transition (EMT). Moreover, the first and second melanoma from the same patient presented a different miRNA profile. Ten miRNAs, including miR-25-3p, 149-5p, 92b-3p, 211-5p, 125a-5p, 125b-5p, 205-5p, 200b-3p, 21-5p and 146a-5p, were further validated in a larger cohort of single and multiple melanoma samples $(\mathrm{N}=47)$. Overall, the Pathway Enrichment Analysis revealed a more differentiated and less invasive status of MPMs. Analyzing our smallRNA seq data, we detected a panel of melanoma-specific miRNA isoforms (isomiRs), which were validated in The Cancer Genome Atlas SKCM cohort. Specifically, we identified hsa-miR-125a-5p|0|-2 isoform as 10-fold over-represented in melanoma and differentially expressed in MPMs. IsomiR-specific target analysis revealed that the miRNA shortening confers a novel pattern of target gene regulations, including genes implicated in melanocyte differentiation and cell adhesion.

Conclusions Overall we provided a comprehensive characterization of the miRNA/isomiRNA regulatory network of multiple primary melanomas, highlighting mechanisms of tumor development and correlating miRNA expression with MPM clinical characteristics.

\section{Background}

Melanoma is a malignant tumor that develops from transformed melanocytes. The incidence of cutaneous melanoma (CM) has been rising constantly in the past several decades, reaching an agestandardized rate of 7, 22 and 70 per 100,000 persons among fair-skinned population in Northern Europe, North America and Australia continent respectively (source: IARC 2018).

CM accounts for $3-5 \%$ of all skin cancers, determining up to $65 \%$ of the deaths ${ }^{19}$. The pathogenesis of $\mathrm{CM}$ is complex and poorly understood. Risk factors include genetic, environmental and phenotypic factors such as ultraviolet (UV) exposure, fair phototypes, multiple dysplastic nevi and a positive family/personal history of $\mathrm{CM}^{12}$. 
Regarding genetic factors, somatic mutations of BRAF gene have been found in almost $40-50 \%$ of sporadic CMs located in body sites with intermittent UV exposure; $15-20 \%$ of the other cases are associated to NRAS mutations and correlated with chronic UV exposure ${ }^{24}$. A small portion of melanomas occurs in acral or mucosal locations and a subset of them are related to KIT and GNAQ mutations ${ }^{7}$. These findings have brought important therapeutic implications and changed the management of CM patients with the development of specific target therapies. Germline mutations instead, can be found in multiple or familial cases of $\mathrm{CM}$. The most frequently described germline mutation is in CDKN2A (cyclin-dependent kinase inhibitor 2A) gene occurring in $8-15 \%$ of subjects diagnosed with multiple primary melanomas (MPMs) without familial history and up to $40 \%$ of patients with hereditary $C M \quad 6,25,40,44$. Mutations in other susceptibility genes such as $C D K 4$ (cyclin-dependent kinase 4), MITF (microphtalmia-associated transcription factor) and POT1 (protection of telomeres 1) are less frequently detected ${ }^{4,14}$. During their lifetime about $8 \%$ of patients with cutaneous melanoma will develop multiple primary melanomas, usually at a young age and within 3 years from the first tumor/diagnosis ${ }^{18}$.

The occurrence of MPMs in the same patient is thought to be related to a personal genetic susceptibility in association with environmental factors. These patients may represent a model of high-risk $\mathrm{CM}$ occurrence. As a matter of fact, it is estimated that a personal history of $\mathrm{CM}$ is a strong risk factor for the development of a subsequent primary $\mathrm{CM}^{18,43}$. The excision of a prior $\mathrm{CM}$ determines a risk up to $8.5 \%$ to develop another CM and the frequency of MPMs is reported to be between 0.2 and $10 \%{ }^{13,25,29}$. The above-reported rates may underestimate the lifetime rates due to limited series of patients and different follow-up periods. Variability may also arise due to differences in environmental factors such as ultraviolet radiation exposure across geographical regions. Among the cases of MPMs, $13-40 \%$ of patients are diagnosed with synchronous lesions (i.e. a subsequent primary CM diagnosed within 3 months from the prior diagnosis), while the remainder develop metachronous lesions ${ }^{1,29,41}$. The risk of a subsequent $\mathrm{CM}$ is highest in the first year following the diagnosis of the primary $\mathrm{CM}$; however, this risk remains increased for at least 20 years ${ }^{1}$. Despite the increased risk of multiple tumor development, there is still an open debate about the prognosis of MPM patients. On one hand, Doubrovsky et al. observer a favorable prognosis in patients with MPM ${ }^{16}$, but a worse prognosis for these patients was recently reported by El Sharouni and colleagues ${ }^{17}$.

Moreover, the frequency of germline mutations in melanoma susceptibility genes (CDKN2A, CDK4, MITF, POT1/ACD/TERF2IP, TERT, BAP1) is lower than expected in MPM patients $5,6,9,25$. Therefore, a better characterization of MPM pathogenesis and biological features is of the outmost importance.

The dysregulation of small noncoding RNAs, specifically microRNAs (miRNAs, 18-22 nucleotides in length), plays a significant role in tumorigenesis, including melanoma onset and progression ${ }^{46}$. MiRNAs regulate multiple and specific target genes, determining an oncogenic or tumor-suppressive function, being implicated in the proliferation, apoptosis and tumor progression. Moreover, miRNA global expression profile faithfully reflects the overall expression profile of normal and pathological cells and 
tissues, with the advantage to be feasible also from formalin-fixed and paraffin-embedded (FFPE) tissues.

In this study, we investigated the global miRNA and isomiRNA expression profile of multiple primary melanomas using an unbiased smallRNA sequencing approach. A comparison of familial/non familial MPM vs. single primary melanoma miRNome was established in order to investigate the possible similarities. Moreover, the evolution of MPM miRNA profile was assessed matching multiple tumors from the same patient.

\section{Methods}

\section{Clinical samples}

A retrospective series of 47 samples from 29 patients was collected. Patients were selected among those referring to the melanoma center of the Dermatology Unit at Bologna University Hospital. The study was approved by Comitato Etico Indipendente di Area Vasta Emilia Centro - CE-AVEC, Emilia-Romagna Region (number 417/2018/Sper/AOUBo). Before study entry, all the patients provided written and voluntary informed consent for inclusion, collection and use of clinical-pathological data and samples and data privacy.

The specimens were classified into three groups: benign nevi, single primary cutaneous melanoma (CM) and multiple primary melanoma (MPM). Group $1(n=3)$, benign nevi of 3 patients with no prior diagnosis of $\mathrm{CM}$ or non-melanoma skin cancer and follow up of at least 10 years. Group $2(n=35)$, MPM samples from 17 patients with prior diagnosis of $\geq 2 \mathrm{CMs}$. 3 out of 17 patients had positive family history of CM (FAM). MPM patients were tested for CDKN2A, MITF and CDK4 genetic alterations and only 1 patient had a mutation in CDKN2A gene (c.249C > A p.His83GIn). Group $3(n=9), 9$ samples from CM patients with no history of prior CMs and a follow-up of at least 10 years.

Tumor and nevi samples were formalin-fixed and paraffin-embedded (FFPE). For each sample, 5/6 tissue sections on glass slides were obtained. One section was stained with hematoxylin-eosin (HE) and examined by an expert pathologist to select the tumor/nevus area, which was grossly dissected before RNA extraction.

\section{RNA extraction}

RNA was isolated from $10 \mu$ m-thick FFPE sections using miRNeasy FFPE kit (Qiagen) according to the manufacturer's instructions. Deparaffinization was performed with xylene followed by an ethanol wash. RNA was eluted in $30 \mu \mathrm{L}$ of RNAse-free water and quantified by absorbance at 260 and $280 \mathrm{~nm}$.

\section{SmallRNA sequencing}

We analyzed 3 benign nevi, 4 single CM, 17 multiple primary or familial melanomas from 8 different patients. The 24 smallRNA libraries were generated using TruSeq Small RNA Library PrepKit v2 (Illumina, 
RS-200-0012/24/36/48), according to manufacturer's indications. Briefly, 35 ng of purified RNA was linked to RNA $3^{\prime}$ and 5 ' adapters, converted into cDNA, and amplified using Illumina primers containing unique indexes for each sample. High Sensitivity DNA kit was adopted for libraries quantification using Agilent Bioanalyzer (Agilent Technologies, California, USA5067-4626) and the 24 DNA libraries were combined in equal amount to generate a libraries pool.

Pooled libraries underwent to size selection employing magnetic beads (Agencourt) and amplicons with a length in the 130-160 bp range, were recovered.

Finally, 20pM of pooled libraries, quantified using the HS-DNA Kit (Agilent) were denatured, neutralized and combined with a Phix control library (standard library normalizator). A $1.8 \mathrm{pM}$ final concentration of pooled libraries (obtained by dilution with a dedicated buffer as described in Illumina protocol guidelines) was obtained and sequenced using NextSeq 500/550 High Output Kit v2 (75 cycles) (Illumina, FC-4042005) on the Illumina NextSeq500 platform.

Raw base-call data were demultiplexed using Illumina BaseSpace Sequence Hub and converted to FASTQ format. After a quality check with FastQC tool, the adapter sequences were trimmed using Cutadapt, which was also used to remove sequences shorter than 16 nucleotides and longer than 30 nucleotides. Reads were mapped using the STAR algorithm. Only reads that mapped unambiguously to the genome (at least 16 nucleotides aligned, with a 10\% mismatch allowed) were used for the analyses. The reference genome consisted in human miRNA sequences from the miRbase 21 database. Raw counts from mapped reads were obtained using the htseq-count script from the HTSeq tools ${ }^{3}$. Counts were normalized using DESeq2 bioconductor package ${ }^{37}$. NGS raw data (FASTQ format) are available through European Nucleotide Archive with the following accession number: PRJEB35819.

\section{Quantitative PCR}

\section{miRCURY LNA Assay}

RNA from 47 samples was converted to cDNA using miRCURY LNA RT kit (Qiagen, cat. no. 339340). Reverse transcription (RT) reaction was performed as follows: $2 \mu \mathrm{L}$ miRCURY RT Reaction Buffer, $4.5 \mu \mathrm{L}$ RNase-free water, $1 \mu \mathrm{L}$ miRCURY RT Enzyme Mix, $0.5 \mu \mathrm{L}$ UniSp6 spike-in, and $2 \mu \mathrm{L}$ template RNA (5 ng/ $\mu \mathrm{L})$. RT cycling protocol consisted in $60 \mathrm{~min}$ at $42^{\circ} \mathrm{C}, 5 \mathrm{~min}$ at $95^{\circ} \mathrm{C}$, and cooling at $4{ }^{\circ} \mathrm{C}$. cDNA samples were stored at-20 ${ }^{\circ} \mathrm{C}$. RT-qPCR was performed using miRCURY LNA SYBER Green PCR kit (Qiagen, cat. no. 339346) and primers from miRCURY LNA miRNA PCR Assays (Qiagen, cat. no. 339306): miR-21-5p (cat. no. YP00204230), miR-25-3p (cat. no. YP00204361), miR-125b-5p (cat. no. YP00205713), miR-146a-5p (cat. no. YP00204688), miR-205-5p (cat. no. YP00204487), miR-149-5p (cat. no. YP00204321), miR-92b$3 p$ (cat. no. YP00204384), miR-200b-3p (cat. no. YP00206071), miR-211-5p (cat. no. YP00204009), miR16-5p (cat. no. YP00205702), miR-125a-5p (cat. no. YP00204339), SNORD44 (cat. no. YP00203902). For each miRNA target, cDNA was diluted at the ratio of 1:80 with the exception of miR-92b-3p, for which cDNA was diluted 1:4. Cycling program consisted in: 10 min at $95^{\circ} \mathrm{C}$ and 2-step cycling (40 cycles) of denaturation (10 s at $95^{\circ} \mathrm{C}$ ), and combined annealing/extension (60 s at $\left.60^{\circ} \mathrm{C}\right)$. Raw Cq values were 
obtained from BioRad CFX software. Interplate calibrators were used to standardize miRNA Cq values across plates. miR-16-5p was selected as reference gene due to its stability across samples in NGS experiment. The calculation of relative expression was performed using $2^{-\Delta C t}$ methods.

\section{miSCRIPT Assay (miR-125a-5p isoforms quantification)}

RNA from 39 samples was reverse transcribed using miSCRIPT HiSpec Buffer from miSCRIPT II RT kit (Qiagen, cat. no. 218161) according to the manufacturer's instructions. Specifically, RT reaction was prepared in a total reaction volume of $10 \mu \mathrm{L}$ with $2 \mu \mathrm{L}$ miScript HiSpec Buffer, $1 \mathrm{ul}$ miScript Nucleics Mix, $4 \mu \mathrm{L}$ RNase-free water, $1 \mu \mathrm{L}$ miScript Reverse Transcriptase Mix, $2 \mu \mathrm{L}$ template RNA ( $5 \mathrm{ng} / \mu \mathrm{L})$. RT cycling protocol consisted in $60 \mathrm{~min}$ at $37^{\circ} \mathrm{C}, 5 \mathrm{~min}$ at $95^{\circ} \mathrm{C}$, and cooling at $4{ }^{\circ} \mathrm{C}$. cDNA samples were stored at$20^{\circ} \mathrm{C}$. RT-qPCR was performed using miSCRIPT SYBER Green PCR kit (Qiagen, cod. no. 218073) and primers from miSCRIPT Primer Assays (Qiagen, cat. no. 218300): miR-125a-5p (cod. no. MS00003423), RNU6 (cod. no. MS00033740). Reaction mix was prepared with $10 \mu \mathrm{L}$ QuantiTect SYBR Green PCR Master Mix, $2 \mu \mathrm{L}$ miScript Universal Primer, $2 \mu \mathrm{L}$ miScript target Primer, $3 \mu \mathrm{L}$ Nase-free water, $3 \mu \mathrm{L}$ cDNA template (1:40).

Cycling program consisted in: $15 \mathrm{~min}$ at $95^{\circ} \mathrm{C}$, and 3-step cycling (40 cycles) of denaturation ( $15 \mathrm{~s}$ at $\left.94^{\circ} \mathrm{C}\right)$, annealing $\left(30 \mathrm{~s}\right.$ at $\left.55^{\circ} \mathrm{C}\right)$ and extension $\left(30 \mathrm{~s}\right.$ at $\left.70^{\circ} \mathrm{C}\right)$. Raw $\mathrm{Cq}$ values were obtained from BioRad CFX software. Small nuclear RNA U6 (RNU6) was used as reference gene. The calculation of relative expression was performed using $2^{-\Delta C t}$ methods.

\section{Quantification of isomiRs}

IsomiRs were identified in our NGS dataset of 24 samples as described in Loher et al. ${ }^{36}$. Briefly, sequence reads were quality trimmed using the cutadapt tool, and mapped unambiguously using SHRIMP2 (PMID: 21278192) to the human genome assembly GRCh38. During the mapping, no insertions or deletions, and at most one mismatch was permitted. IsomiRs were identified as done previously 36,52 .

For TCGA isomiR analysis, short RNA-seq Aligned BAM files were downloaded from the Genomic Data Commons Data Portal (https://portal.gdc.cancer.gov/) for all 32 cancer types. IsomiR profiles were generated using the same approach as described in Loher et al. ${ }^{36}$

To simplified the labeling of the isomiRs, we used the annotation system developed by Loher et al. This nomenclature specifies the name of the canonical miRNA, the start site ( 5 ' end) of the isomiR compared to the canonical miRNA sequence in miRBase, the end-site ( 3 ' end) and the eventual insertion of uracil. In particular, to annotate the start and end site of an isomiR, a negative (-) or positive sign (+) followed by the number of nucleotides is used to indicate how many nucleotides the isomiRs terminus has, when compared to the canonical miRNA sequence. Zero indicate the same terminus of the canonical miRNA sequence.

We quantified isomiR abundances in reads per million (RPM). Only reads that passed quality trimming and filtering and could be aligned exactly to miRNA arms were used in the denominator of this 
calculation.

IsomiR targets were predicted using the RNA22 algorithm ${ }^{35}$ and targets were allowed to be present in the 5'UTR, CDS, and 3'UTR of the candidate mRNA. We selected only those targets that had a $p$-value $<0.01$ and a predicted binding energy <-16 while also allowing G:U wobbles and bulge's within the seed region.

\section{Statistical analysis}

Normalized sequencing data were imported and analyzed in Genespring GX software (Agilent Technologies). Differentially expressed miRNAs were identified using a fold change $>1.5$ filter and moderated t-test (FDR 5\% with Benjamini-Hochberg correction) in CM vs. MPM comparison and foldchange $>1.2$ and paired t-test $(p<0.05)$ in 1st vs. 2nd MPM comparison. Cluster Analysis, was performed using Manhattan correlation as a similarity measure. Principal Component Analysis was performed on 24 samples using all human miRNAs detected by NGS analysis ( $n=1629)$.

Graphpad Prism 6 (GraphPad Software) was used for statistical analyses. Group comparison was performed using unpaired t-test, when data had a normal distribution, with or without Welch's correction according to the significance of the variance test. Data that did not present a normal distribution were compared using Mann-Whitney non-parametric test.

Association of gene expression with overall survival in TCGA SKCM cohort, was obtained using Oncolnc website (http://www.oncolnc.org), logrank test was used to calculate the p-value.

\section{Pathway Analysis}

Pathway and network analysis of differentially expressed miRNAs, miR-125a-5p isoforms and their targets was investigated using the web-based software MetaCore (GeneGo, Thomson Reuters). A p value of 0.05 was used as a cut off to determine significant enrichment.

\section{Results}

\section{Patient Characteristics}

Demographic, clinical and pathological features of 29 patients are summarized in Table 1. A total number of 16 males and 13 females were included, with a mean age at first diagnosis of 59 years for single primary melanomas and 53 years for multiple primary melanomas. Nine patients had single cutaneous melanoma and 10 years of follow up; 17 developed more than one primary melanoma in an average time of 33 months (range 3-98). MPM patients were tested for germline genetic alterations in CDKN2A, CDK4 and MITF gene ${ }^{15}$ and only one patient was found to have a germline CDKN2A mutation (c.249C > A p.His83GIn exon 2) of unknown clinical significance. Melanoma specimens were examined by two dermato-pathologists. 
Table 1

Patient characteristics

\begin{tabular}{llll}
$\begin{array}{l}\text { Benign } \\
\text { Nevi (BN) }\end{array}$ & $\begin{array}{l}\text { Cutaneous } \\
\text { Melanoma (CM) }\end{array}$ & \multicolumn{2}{l}{$\begin{array}{l}\text { Multiple Primary } \\
\text { (MPM) }\end{array}$} \\
\cline { 3 - 5 } & $\begin{array}{lll}\text { MPM } \\
\text { 1st }\end{array}$ & $\begin{array}{l}\text { MPM } \\
\text { 2nd }\end{array}$ & $\begin{array}{l}\text { MPM } \\
\text { 3rd }\end{array}$
\end{tabular}

Gender (n;\%)

Male

$0(0 \%)$

$3(33.3 \%)$

$13(76.5 \%)$

Female

$3(100 \%) \quad 6(66.7 \%)$

$4(23.5 \%)$

Total

3

9

17

Histology $(\mathrm{n} ; \%)$

Compound melanocytic nevus

$2(66.7 \%)$

Dermal nevus

$1(33.3 \%)$

Superficial spreading melanoma

$4(44.5 \%)$

15

$(88.2 \%) \quad(94.1 \%) \quad(100 \%)$

Superficial spreading melanoma

with vertical growth phase

$3(33.3 \%)$

1

$(5.9 \%)$

Nodular melanoma

$2(22.2 \%)$

1

$(5.9 \%)$

Nevus-associated melanoma

0

1

$(5.9 \%)$

Age at first diagnosis (n;\%)

$\begin{array}{llll}<50 & - & 7(77.8 \%) & 8(47 \%) \\ \geq 50 & - & 2(22.2 \%) & 9(53 \%) \\ \text { Mean (range) } & - & 59(29-85) & 53(30-80)\end{array}$

Localization (n;\%)

Trunk

$3(100 \%) \quad 6(66.7 \%)$

13

$(76.5 \%) \quad(82.4 \%) \quad(100 \%)$

Limbs

$2(22.2 \%)$

$\begin{array}{ll}3 & 3 \\ (17.6 \%) & (17.6 \%)\end{array}$

Head and neck

$1(11.1 \%)$

1

$(5.9 \%)$

Breslow thickness $(n ; \%)$

$<0.8 \mathrm{~mm}$

$1(11.1 \%)$

$\begin{array}{lll}12 & 17 & 1 \\ (70.6 \%) & (100 \%) & (100 \%)\end{array}$ 


\begin{tabular}{|c|c|c|c|c|c|}
\hline & \multirow[t]{2}{*}{$\begin{array}{l}\text { Benign } \\
\text { Nevi (BN) }\end{array}$} & \multirow[t]{2}{*}{$\begin{array}{l}\text { Cutaneous } \\
\text { Melanoma (CM) }\end{array}$} & \multicolumn{3}{|c|}{$\begin{array}{l}\text { Multiple Primary Melanoma } \\
\text { (MPM) }\end{array}$} \\
\hline & & & $\begin{array}{l}\text { MPM } \\
\text { 1st }\end{array}$ & $\begin{array}{l}\text { MPM } \\
\text { 2nd }\end{array}$ & $\begin{array}{l}\text { MPM } \\
\text { 3rd }\end{array}$ \\
\hline$\geq 0.8 \mathrm{~mm}$ & - & $8(88.9 \%)$ & $\begin{array}{l}5 \\
(29.4 \%)\end{array}$ & $0(0 \%)$ & - \\
\hline \multicolumn{6}{|c|}{ Family history of melanoma (n;\%) } \\
\hline Yes & - & - & \multicolumn{3}{|c|}{$3(17.6 \%)$} \\
\hline No & - & - & \multicolumn{3}{|c|}{14 (82.4\%) } \\
\hline
\end{tabular}

\section{The microRNA profile of multiple primary melanoma}

The global miRNA profile of 17 multiple primary melanomas, obtained from 8 patients, was analyzed using a smalIRNA sequencing approach. For each MPM patient we analyzed the first and second primary tumor, and for one case also a third one. Three patients had a family history of melanoma. We compared the global miRNA profile of MPM toward that of 4 single melanomas and 3 benign nevi. From the smallRNA sequencing data, we identified 1629 mature miRNAs expressed in melanoma and nevus cells. The unsupervised Principal Component Analysis (PCA) of all miRNAs and all samples $(n=24)$ revealed that familial and non-familiar multiple primary melanomas have a greatly overlapping miRNA profile (Fig. 1A), which is different from single cutaneous melanoma (CM) and benign nevi (BN). Indeed, a statistical comparison between familial and non-familial MPMs did not provide any significant result. Therefore, we considered familial and non-familial melanomas as a unique group in all subsequent analyses. From the PCA we can already observe that MPMs displayed a miRNA profile more similar to benign nevi than CMs. When we compared multiple and single melanoma tumors, we obtained a markedly different miRNA expression profile and a list of 22 miRNAs differentially expressed (adjusted $p$ $<0.05$, Table 2), which are represented with a Volcano plot in Fig. 1B. Cluster analysis of these samples based on the expression of the 22 differentially expressed miRNAs confirmed the separation between single and multiple tumors Fig. 1C. The MPM group was constituted by the paired first and second tumors (and one additional tumor in one case) developed by the same patient over the years (Table 1). Comparing the miRNA profile of these two groups using a paired statistical analysis, miRNAs that characterize the second tumors, usually thinner and less aggressive than the first melanoma given their early diagnosis, were identified. Despite the similarities between the two matching MPMs, a variation in miRNA expression was observed (Fig. 1B). Specifically, thirty-seven miRNAs were differentially expressed between the first and second MPM (paired t-test, $p<0.05$, Table 3 ) and a significant separation was obtained applying the cluster analysis (Fig. 1D). 
Table 2

- List of microRNAs differentially expressed in multiple vs. primary melanoma

\begin{tabular}{|c|c|c|c|}
\hline microRNA & adjusted p-value & Regulation MPM vs. CM & Fold change \\
\hline hsa-miR-25-3p & 0.0084 & down & -2.2 \\
\hline hsa-miR-3614-5p & 0.0135 & down & -3.2 \\
\hline hsa-let-7i-5p & 0.0153 & down & -2.7 \\
\hline hsa-miR-181a-3p & 0.0153 & down & -2.7 \\
\hline hsa-miR-21-3p & 0.0153 & down & -4.7 \\
\hline hsa-miR-584-5p & 0.0167 & down & -6.3 \\
\hline hsa-miR-149-5p & 0.0205 & up & 3.2 \\
\hline hsa-miR-21-5p & 0.0205 & down & -4.3 \\
\hline hsa-miR-29a-3p & 0.0205 & down & -1.7 \\
\hline hsa-miR-146a-5p & 0.0250 & down & -4.8 \\
\hline hsa-miR-651-5p & 0.0275 & down & -1.7 \\
\hline hsa-miR-125b-2-3p & 0.0314 & up & 2.1 \\
\hline hsa-miR-15a-5p & 0.0314 & down & -2.3 \\
\hline hsa-miR-99a-5p & 0.0314 & up & 2.4 \\
\hline hsa-miR-941 & 0.0318 & down & -1.8 \\
\hline hsa-miR-340-5p & 0.0341 & down & -2.4 \\
\hline hsa-miR-532-5p & 0.0341 & down & -2.1 \\
\hline hsa-miR-205-5p & 0.0344 & up & 2.1 \\
\hline hsa-miR-106b-3p & 0.0347 & down & -2.3 \\
\hline hsa-miR-181c-5p & 0.0360 & down & -2.0 \\
\hline hsa-miR-125b-5p & 0.0398 & up & 2.4 \\
\hline hsa-miR-132-3p & 0.0450 & down & -1.8 \\
\hline
\end{tabular}


Table 3

List of microRNAs differentially expressed in paired multiple melanomas from the same patient

\begin{tabular}{|c|c|c|c|}
\hline microRNA & p-value (paired) & Regulation MPM vs. CM & Fold change \\
\hline hsa-let-7d-3p & 0.0174 & down & -1.8 \\
\hline hsa-let-7e-5p & 0.0153 & down & -2.0 \\
\hline hsa-miR-1226-3p & 0.0345 & down & -3.1 \\
\hline hsa-miR-1248 & 0.0423 & up & 3.4 \\
\hline hsa-miR-1249 & 0.0338 & down & -1.8 \\
\hline hsa-miR-125a-5p & 0.0063 & down & -1.9 \\
\hline hsa-miR-1269b & 0.0254 & down & -2.0 \\
\hline hsa-miR-145-3p & 0.0313 & down & -1.7 \\
\hline hsa-miR-149-5p & 0.0157 & down & -1.7 \\
\hline hsa-miR-200b-3p & 0.0134 & down & -1.8 \\
\hline hsa-miR-223-3p & 0.0458 & up & 1.8 \\
\hline hsa-miR-224-5p & 0.0135 & down & -1.8 \\
\hline hsa-miR-2392 & 0.0362 & up & 2.3 \\
\hline hsa-miR-320b & 0.0243 & down & -2.0 \\
\hline hsa-miR-328-3p & 0.0103 & down & -1.9 \\
\hline hsa-miR-330-5p & 0.0060 & down & -1.5 \\
\hline hsa-miR-3607-5p & 0.0308 & up & 3.1 \\
\hline hsa-miR-3609 & 0.0028 & up & 2.5 \\
\hline hsa-miR-365a-5p & 0.0398 & down & -1.6 \\
\hline hsa-miR-375 & 0.0166 & down & -1.8 \\
\hline hsa-miR-4286 & 0.0071 & up & 1.9 \\
\hline hsa-miR-433-3p & 0.0019 & down & -2.2 \\
\hline hsa-miR-4423-3p & 0.0090 & down & -2.0 \\
\hline hsa-miR-4466 & 0.0190 & up & 2.0 \\
\hline hsa-miR-505-3p & 0.0194 & down & -1.8 \\
\hline hsa-miR-6511b-3p & 0.0299 & down & -2.0 \\
\hline
\end{tabular}




\begin{tabular}{|llll|}
\hline microRNA & p-value (paired) & Regulation MPM vs. CM & Fold change \\
\hline hsa-miR-671-3p & 0.0431 & down & -1.6 \\
\hline hsa-miR-7641 & 0.0129 & up & 2.1 \\
\hline hsa-miR-8058 & 0.0334 & down & -2.2 \\
\hline hsa-miR-877-5p & 0.0160 & down & -2.1 \\
\hline hsa-miR-887-3p & 0.0441 & up & 2.0 \\
\hline hsa-miR-92a-1-5p & 0.0464 & down & -1.6 \\
\hline hsa-miR-92b-3p & 0.0100 & down & -1.6 \\
\hline hsa-miR-96-5p & 0.0062 & down & -2.6 \\
\hline hsa-miR-98-3p & 0.0246 & down & -1.8 \\
\hline hsa-miR-99a-3p & 0.0342 & up & 1.6 \\
\hline hsa-miR-99b-5p & 0.0054 & down & -1.6 \\
\hline
\end{tabular}

Validation of microRNA differential expression in single and multiple primary melanomas and paired primary tumors from the same patient

Nine miRNAs were selected for an independent technical validation using quantitative RT-PCR in 47 novel samples including BN, CM and 1st and 2nd MPM. Specifically, we included the miRNAs differentially expressed between CM and MPM (miR-21-5p, miR-25-3p, miR-125b-5p, miR-146a-5p, miR-205-5p, miR149-5p) and others between the first (MPM 1st) and second (MPM 2nd) melanoma within the same MPM patient (miR-149-5p, miR-92b-3p, miR-200b-3p, miR-125a-5p).

According to the smallRNA NGS results, an upregulated expression of miR-21-5p, miR-25-5p, miR-146a$5 p$, and a downregulated expression of miR-125b-5p, miR-149-5p and miR-205-5p in CM compared to MPM were expected. In MPM samples, all selected miRNAs are upregulated in the MPM 2nd compared to MPM 1st. In the validation experiment, we included also miR-211-5p, considering that it is a melanocyte specific miRNA, whose genetic locus is located inside melastatin gene and whose expression is particularly high in nevi. The expression of this miRNA was higher in BN, with borderline statistical significance when compared to CM or MPM in our NGS data.

The validation was performed in a cohort of 29 patients, as described in Table 1, using miR-16-5p as a reference gene due to its invariant expression in NGS data. Expression distributions of selected miRNAs in benign nevi, cutaneous melanoma and multiple primary melanoma samples are represented in Fig. 2 . The significant upregulation of miR-21-5p in CM vs. BN and MPM was confirmed. Moreover, a statistically significant miR-25-3p downregulation in CM and MPM compared to BN was observed. miR-146a-5p resulted downregulated in MPM compared to both BN and CM. For miR-125b-5p, a similar expression level in $\mathrm{CM}$ and $\mathrm{BN}$, and a trend toward increased expression in MPM was obtained. A significant 
upregulation of miR-200b-3p and miR-205-5p was observed in MPM. A similar trend can be observed for miR-149-5p. As expected, the melanocyte specific, MITF-regulated miR-211-5p is progressively downregulated in multiple and single melanomas (Fig. 2).

The differential expression of miR-149-5p, miR-92b-3p, miR-200b-3p, miR-205-5p between paired first and second melanomas from the same patient is represented in Fig. 3. A significant upregulation for miR-1495p, miR-92b-3p, miR-205-5p and miR-200b-3p in MPM 2nd tumor was confirmed in this larger group of samples.

\section{Functional annotation of multiple primary melanoma miRNA signature}

The list of 22 miRNAs differentially expressed in multiple $v s$. single melanomas, was uploaded in Metacore software (Clarivate Analytics) to identify both the pathways that are significantly regulated by these miRNAs (Supplementary Table 1, Additional file 1) and the most significant miRNAs/targets networks (Supplementary Fig. 1A, Additional file 2).

Multiple primary melanomas were found to have a higher expression or miR-200 family, miR-205-5p and miR-149-5p compared to single CM and even nevi (Fig. 2 and Fig. 4). These microRNAs target ZEB1/TCF8 and ZEB2/SIP1 genes, and by doing so they inhibit the epithelial-mesenchymal transition (EMT) pathway. This pathway therefore appears to be specifically activated in single melanomas (Fig. 4).

From MetaCore network analysis, three hub genes (TLR4, ITGA6 and BTG2) were identified as targeted by multiple miRNAs, either up- or down-regulated in multiple melanomas. When we assessed the association of TLR4, ITGA6 and BTG2 gene expression with melanoma prognosis, we observed that their higher expression (median cutoff) was significantly associated with a worse overall survival in TCGA SKCM cohort of 458 samples (Supplementary Fig. 1B, Additional file 2).

IsomiRNA analysis revealed that miR-125a-5p isoforms are dysregulated in multiple primary melanoma

Interestingly, miR-125a-5p differential expression in MPM was not confirmed by qPCR technology and we wondered about a possible explanation. We observed that the reads generated by the smallRNA sequencing experiment and attributed to mature miR-125a-5p following the standard matching pipeline were actually shorter by 1 , or most frequently 2 nucleotides (lack of GA at the $3^{\prime}$ end) in all samples (Supplementary Fig. 2A, Additional file 3). Although miRBase database reports a unique mature sequence for each miRNA, the so called canonical form, many evidences from deep sequencing experiments suggest that miRNAs have frequent modifications in length and sequence in human tissues. These miRNA isoforms are called isomiRs ${ }^{11}$.

We analyzed the isomiR expression level in all single and multiple primary CMs from our NGS experiments. We found 90 miRNAs with sequence and length heterogeneity, generating 324 different isomiRs, and 40 canonical microRNAs without any isomiR. In addition, we found 40 isomiRs named "orphan", because their canonical miRNA sequences could not be detected. For each isomiR, we 
calculated the average expression in melanoma samples and the ratio between each isomiR and its canonical miRNA. Finally, we obtained a panel of 17 miRNAs whose isoforms are 3- to 10-fold more abundant in melanoma than their canonical form (Table 4). Among them, hsa-miR-125a-5p|0|-2 isoform was differentially expressed in multiple vs. single primary melanomas and between the first and second tumor of the same patient (paired t-test $P=0.0006$ ). Unusually, miR-125a-5p canonical and 3 ' shorter isoforms show an opposite expression trend in nevi, single and multiple primary melanomas (Fig. $5 \mathrm{~A}$ ). 
Table 4

IsomiRs most represented in melanoma

\begin{tabular}{|c|c|c|c|c|}
\hline IsomiR & Type & $\begin{array}{l}\text { IsomiR } \\
\text { expression } \\
\text { (mean) }\end{array}$ & $\begin{array}{l}\text { Canonical miRNA } \\
\text { expression (mean) }\end{array}$ & $\begin{array}{l}\text { Ratio } \\
\text { isomiR/canonical } \\
\text { miRNA }\end{array}$ \\
\hline $\begin{array}{l}\text { hsa-miR-141- } \\
3 p|0|-1\end{array}$ & $\begin{array}{l}\text { end-site } \\
\text { isomiR }\end{array}$ & 200.33 & 8.65 & 23.15 \\
\hline $\begin{array}{l}\text { hsa-miR-222- } \\
3 p|0|+3\end{array}$ & $\begin{array}{l}\text { end-site } \\
\text { isomiR }\end{array}$ & 154.64 & 9.05 & 17.08 \\
\hline $\begin{array}{l}\text { hsa-miR-30a- } \\
5 p|0|+2\end{array}$ & $\begin{array}{l}\text { end-site } \\
\text { isomiR }\end{array}$ & 211.95 & 13.33 & 15.90 \\
\hline $\begin{array}{l}\text { hsa-miR-125a- } \\
5 p|0|-2\end{array}$ & $\begin{array}{l}\text { end-site } \\
\text { isomiR }\end{array}$ & 765.69 & 56.46 & 13.56 \\
\hline $\begin{array}{l}\text { hsa-miR-30d- } \\
5 \mathrm{p}|0|+2\end{array}$ & $\begin{array}{l}\text { end-site } \\
\text { isomiR }\end{array}$ & 416.87 & 41.32 & 10.09 \\
\hline $\begin{array}{l}\text { hsa-miR-10b- } \\
5 p|0|-1\end{array}$ & $\begin{array}{l}\text { end-site } \\
\text { isomiR }\end{array}$ & 2950.88 & 357.98 & 8.24 \\
\hline $\begin{array}{l}\text { hsa-miR-27a- } \\
3 p|0|-1\end{array}$ & $\begin{array}{l}\text { end-site } \\
\text { isomiR }\end{array}$ & 167.78 & 30.55 & 5.49 \\
\hline $\begin{array}{l}\text { hsa-miR-30a- } \\
5 p|0|-2\end{array}$ & $\begin{array}{l}\text { end-site } \\
\text { isomiR }\end{array}$ & 72.12 & 13.33 & 5.41 \\
\hline $\begin{array}{l}\text { hsa-miR-222- } \\
3 p|0|+4\end{array}$ & $\begin{array}{l}\text { end-site } \\
\text { isomiR }\end{array}$ & 46.98 & 9.05 & 5.19 \\
\hline $\begin{array}{l}\text { hsa-miR-19b- } \\
3 p|0|-1\end{array}$ & $\begin{array}{l}\text { end-site } \\
\text { isomiR }\end{array}$ & 52.06 & 11.59 & 4.49 \\
\hline $\begin{array}{l}\text { hsa-miR-30c- } \\
5 p|0|+1\end{array}$ & $\begin{array}{l}\text { end-site } \\
\text { isomiR }\end{array}$ & 151.53 & 34.28 & 4.42 \\
\hline $\begin{array}{l}\text { hsa-miR-26b- } \\
5 p|0|+1\end{array}$ & $\begin{array}{l}\text { end-site } \\
\text { isomiR }\end{array}$ & 172.47 & 39.32 & 4.39 \\
\hline $\begin{array}{l}\text { hsa-miR-222- } \\
3 p|0|+2\end{array}$ & $\begin{array}{l}\text { end-site } \\
\text { isomiR }\end{array}$ & 36.83 & 9.05 & 4.07 \\
\hline $\begin{array}{l}\text { hsa-miR-10a- } \\
5 p|0|-1\end{array}$ & $\begin{array}{l}\text { end-site } \\
\text { isomiR }\end{array}$ & 636.58 & 184.52 & 3.45 \\
\hline $\begin{array}{l}\text { hsa-miR-30a- } \\
5 p|0|+1\end{array}$ & $\begin{array}{l}\text { end-site } \\
\text { isomiR }\end{array}$ & 45.68 & 13.33 & 3.43 \\
\hline $\begin{array}{l}\text { hsa-miR-30d- } \\
5 p|0|+1\end{array}$ & $\begin{array}{l}\text { end-site } \\
\text { isomiR }\end{array}$ & 140.07 & 41.32 & 3.39 \\
\hline $\begin{array}{l}\text { hsa-miR-200b- } \\
3 p|0|+1\end{array}$ & $\begin{array}{l}\text { end-site } \\
\text { isomiR }\end{array}$ & 44.86 & 13.26 & 3.38 \\
\hline
\end{tabular}


We studied two different technical approaches for miRNA quantification based on qPCR (miRCURY LNA and miSCRIPT, both by Qiagen), to selectively quantify miR-125a-5p isoforms in all samples and validate the NGS data. Specifically, we used miR-125a-5p miRCURY LNA assay (Exiqon/Qiagen) for the quantification of the canonical, 24nt-long isoform (Supplementary Fig. 2B, Additional file 3). Results revealed a lack of variation of this mature isoform between single and multiple melanomas, and a higher expression in the first vs. second melanoma (Fig. 5B,C). To quantify the miR-125a-5p 22nt-long isoform, we selected the miSCRIPT assay by Qiagen. The assay can quantify both the long and short isoforms of miR-125a-5p due to the use of a universal 3' primer for miRNA amplification. Given the high predominance of the short isoform in our NGS data, we assumed this assay could provide a bona fide quantification of the short 22nt-long isoform (Supplementary Fig. 2B, Additional file 3). As expected, an increase in miR-125a-5p levels in MPMs vs. CMs and in the second tumor from the same patient was observed (Fig. 5B,C). We examined the expression of hsa-miR-125a-5p|0|-2 and 0|0 (WT) isoforms across TCGA tumor types and discovered an overall higher expression of the shorter form in human cancers and a specifically altered ratio of the two forms in SKCM (cutaneous melanoma cohort), which shows the largest variation (Fig. 6).

\section{miR-125a-5p 3' isomiR regulate novel target genes and pathways}

We run a bioinformatics analysis to predict the impact of miR-125a-5p 3' isomiR generation in target gene binding. We used RNA22 algorithm to obtain the list of putative pairing sites for miR-125a-5p canonical form (WT, $N=1342$ ) and its 3 ' isoform miR-125a-5p|0|-2 (ISO, $N=971$ ) (Supplementary Table 2, Additional file 4). The predicted target genes were submitted for functional annotation to MetaCore website. The shorter miR-125a-5p isoform lose the ability to bind a fraction of canonical miR-125a-5p|0|0 targets. Significantly enriched pathways and networks were identified for common and specific targets of canonical miR-125a-5p canonical miR-125a-5p|0|0 and its 3' isomiR miR-125a-5p|0|-2 (Supplementary Table 3, Additional file 5) revealing a loss of targeting for miR-125a-5p|0|-2 of genes involved in nervous system development, neurogenesis and neuronal differentiation. IsomiR miR-125a-5p|0|-2 no longer targets key genes involved in cell adhesion and migration (Ephrin receptors, Netrin 1) or intracellular signaling (PIK3C2B).

\section{Discussion}

The risk of melanoma development is influenced by environmental and genetic factors. Families with history of melanoma could have a germline mutation that confers hereditary susceptibility, and this is particularly demonstrated in families where more members develop multiple primary melanomas. In 1968, Lynch and Krush described the familial atypical multiple mole-melanoma (FAMMM syndrome) which encompasses an association between pancreatic cancer, multiple nevi, and melanoma ${ }^{38}$. In the 70 s Clark described a similar phenotype, the B-K mole syndrome, consisting of familial melanoma in the setting of numerous atypical nevi. In the early 1990's, germline mutations in the cell cycle gene, p16 (CDKN2A), were reported among a subset of FAMMM kindreds. Nowadays, most studies report a very low prevalence of CDKN2A /CDK4 in familial or multiple melanoma patients, especially in the Southern 
Europe countries ${ }^{9}$. Though MPM patients often report similar sun exposure experiences, the high percentage of atypical nevi in these patients and their family members, the frequent family history of melanoma, as well as the early onset of melanoma (young age) suggest that predisposing factors for the development of multiple melanomas are involved. Regardless of family history, they are reported also cases of multiple primary melanoma in individuals without familial history of melanoma. In these cases, germline mutations in melanoma predisposing genes are rarely detected. Therefore, it is evident that some other genetic or epigenetic factor is active in multiple primary melanoma to fuel multiple events of melanocytic transformation.

In this study, we provide the first comprehensive molecular characterization of MPMs by assessing their miRNome with a smallRNA sequencing approach. The global microRNA expression reflects the mRNA expression of cells and tissues, with the advantage of being assessable in FFPE tissues. This analysis revealed a specific expression pattern of multiple melanoma tumors when compared to single cutaneous melanoma. MPM miRNome is more similar to benign nevi, thus suggesting a less aggressive and more differentiated phenotype. We validated a panel of microRNAs in additional samples, including also multiple tumors from the same patient, obtaining a panel of microRNAs differentially expressed in tumors from the same patient.

Prognosis of patients with multiple primary CM is an old, but still open, question ${ }^{27}$. Many studies have attempted to address this issue and results are still controversial, with studies stating that developing multiple melanomas is associated with worse prognosis or the opposite $27,48,54$. Recently, Grossman et al. (2018) revealed the potential reasons for these controversies by analyzing with proper multivariate statistical analysis the Surveillance, Epidemiology, and End Results (SEER) data using a single matching method ${ }^{21}$ and demonstrating no substantial difference among single and multiple melanoma patients.

We provide here evidence that MPMs, from a biological point of view, have a less invasive phenotype as pointed out by the main regulatory pathways activated in these tumors, thus providing further elements of discussion to support MPM less aggressive evolution. It is worth mentioning that microRNAs known to inhibit epithelial-mesenchymal transition (e.g. miR-200 family, miR-205, miR-149) are more expressed in multiple primary melanoma compared to single melanoma. Tumor cells promote EMT to escape from the microenvironment and migrate to a new location to develop metastasis ${ }^{22}$. The acquisition of a mesenchymal phenotype promotes the production of extracellular matrix proteins, the resistance to apoptosis, the invasiveness and the migration ${ }^{30}$. EMT results from the loss of cell-to cell junctions, induced by the loss of E-cadherin; the process is mediated by transcription factors, including SNAIL, SLUG, SIP1, and E2A, and affected by regulatory proteins such as TGF $\beta$, EGF, PDGF, ERK/MAPK, $\mathrm{PI} 3 \mathrm{~K} / \mathrm{AKT}$, SMADS, RHOB, $\beta$-catenin, LEF, RAS, C-FOS, integrins $\beta 4$ and integrin a ${ }^{53}$., EMT has been reported in melanoma cells, despite their origin from neural crest-derived melanocytes. In fact, EMT promotes the metastatic phenotype of malignant melanocytes ${ }^{28}$. Moreover, melanocytes express $\mathrm{E}$ cadherin, which mediates the adhesion between melanocytes and keratinocytes ${ }^{51}$. Many studies described the loss of E-cadherin in melanoma ${ }^{32,49}$, and $\mathrm{CDH} 1$ ectopic expression was associated with 
the downregulation of adhesion receptors, such as MCAM/MUC18 and $\beta 3$ integrin subunit, resulting in suppression of melanoma cells invasion ${ }^{26}$. Hao et al. observed a switch from E-cadherin to N-cadherin expression in melanoma progression, a process regulated by PI3K and PTEN through TWIST and SNAIL 23.

Consistently, we examined the main cellular hubs regulated by MPM specific miRNAs and discovered that they are centered in TLR4, ITGA6 and BTG2 proteins. MicroRNAs regulating these hubs are mostly downregulated in MPMs and high expression of these three genes is associated with a more favorable prognosis in TCGA SKCM cohort.

Integrin a6 (ITGA6), also known as CD49f, is a transmembrane glycoprotein adhesion receptor that mediates cell-matrix and cell-cell interactions. ITGA6 was identified and described as an important stem cell biomarker. Indeed, it is the only common gene expressed in embryonic stem cells, neural stem cells and hematopoietic stem cells ${ }^{28,45}$. It is also expressed in more than 30 stem cell populations, including cancer stem cells ${ }^{31}$. ITGA6 can combine with other integrins such as integrin beta 1 and integrin beta 4 to form respectively integrin VLA- 6 and TSP180. The role of ITGA6 in melanoma is not clear but our observation point toward its upregulation in MPMs upon miR-25 and 29 downregulation.

BTG2 is part of the anti-proliferative BTG/TOB family and its expression is p53-dependent ${ }^{47}$. This protein is involved in several cellular processes, including cell cycle regulation, DNA damage repair, cell differentiation, proliferation and apoptosis. However, its role is often cell-type dependent ${ }^{39}$. In fact, BTG2 inhibits proliferation and migration, acting as a tumor suppressor protein, in gastric cancer cells ${ }^{59}$ and in lung cancer cells ${ }^{57}$, while in bladder cancer it promotes cancer cell migration ${ }^{55}$. In B16 melanoma cells it was shown that miR-21 promotes a metastatic behavior through the downregulation of many tumor suppressor proteins, including PTEN, PDCD4 and BTG2 ${ }^{58}$. In MPMs, we observe the downregulation of several miRNAs targeting BTG2, including miR-21-5p, 146a-5p, 132-3p, 15a-5p. Therefore, an upregulation of BTG2 is to be expected.

Toll-like Receptor 4 (TLR4) belongs to TLR family and plays an important role in inflammation and cancer. TLR4 protein is expressed at very low levels in melanoma cells in vivo (Human protein atlas) but its activation has been reported to promote an inflammatory microenvironment and tumor progression in vitro ${ }^{20}$. In addition, TLR4 is associated with induction of proliferation and migration of melanoma cells 50. TLR4 plays an important role in melanoma also because it interacts with TRIM44, a negative prognostic factor in melanoma. In particular, TRIM44 binds and stabilizes TLR4 leading to the activation of AKT/mTOR signaling, which results in EMT promotion ${ }^{56}$. This biological role for TLR4 in melanoma is partially in contrast with our observation of a better survival in melanoma patients with higher TLR4 levels.

Finally, we extended our molecular investigation to miRNA isoforms that were most abundant in our samples. According to the recent observation that miRNA isoforms can discriminate human cancers ${ }^{52}$, 
we detected a relevant number of miRNA variants in our dataset of single and multiple melanomas. A specific isoform of miR-125a-5p, lacking 2 nucleotides at the 3'end, was detected as differentially expressed in MPMs. This isoform is highly abundant in melanoma, as we confirmed by analyzing its levels across 32 tumor types from TCGA database; and the ratio between miR-125a-5p isoform and canonical form is the broader in TCGA SKCM tumors (range 0.1-1100 times) and 2-6 logs more abundant in nevi and melanomas in our study. Moreover, we detected a specific dysregulation of the isoform, but not the canonical form, in multiple melanomas. Bioinformatic analyses revealed that miR$125 a-5 p$ shorted isoform loses the ability to target and regulate a group of genes specifically involved in cell adhesion and cell differentiation. Particularly relevant seems to be the lack of regulation of genes involved in neuronal differentiation. Indeed, miR-125a is the human ortholog of lin-4, the very first miRNA identified in C. Elegans in $1993^{34}$. In mammals, miR-125 is expressed in embryonic stem cells and promote cell differentiation. Specifically, miR-125 has a specific role in adult nervous system development and neuronal differentiation ${ }^{10,33}$. The imbalance between major miR-125 isoforms in melanocytes could reflect a major role for miR-125 in melanocyte development and differentiation from the neural crest ${ }^{42}$, differentiating this lineage from other common ancestor cells. A role that is consequently reflected in melanoma development and progression.

\section{Conclusions}

Overall, we provide here a comprehensive characterization of microRNA/isomiRNA dysregulation and regulatory network in single and multiple primary melanomas. The pattern of miRNA alterations supports a less aggressive phenotype of multiple primary melanomas, whilst isomiR-125a-5p levels proved to be enriched in melanoma and differentially expressed in MPMs, thus confirming the relevance of small noncoding RNA alterations in this fascinating - but poorly studied - melanoma subtype. Our observations about non-random dysregulation of specific miRNA isoforms in melanoma pose the basis for additional functional studies.

\section{List Of Abbreviations}

cutaneous melanoma (CM)

multiple primary melanomas (MPM)

benign nevi (BN)

miRNA isoforms (isomiRs)

ultraviolet (UV)

CDKN2A (cyclin-dependent kinase inhibitor 2A)

CDK4 (cyclin-dependent kinase 4)

Page 20/33 
MITF (microphtalmia-associated transcription factor)

POT1 (protection of telomeres 1)

microRNAs (miRNAs)

formalin-fixed and paraffin-embedded (FFPE)

hematoxylin-eosin (HE)

Reverse transcription (RT)

Small nuclear RNA U6 (RNU6)

reads per million (RPM)

Principal Component Analysis (PCA)

epithelial-mesenchymal transition (EMT)

Toll-like Receptor 4 (TLR4)

\section{Declarations}

\section{Ethics approval and consent to participate}

The study was conducted in accordance with the Declaration of Helsinki, and the protocol was approved by the Ethics Committee Center Emilia-Romagna Region - Italy (417/2018/Sper/AOUBo).

\section{Consent for publication}

All authors of the manuscript have read and agreed to its content.

\section{Availability of data and material}

Next Generation Sequencing raw data (FASTQ format) are available through European Nucleotide Archive (ENA) with the following accession number: PRJEB35819.

\section{Competing interests}

The authors declare no competing interests

\section{Funding}

This work was supported by the Italian Association for Cancer Research (AIRC) funds to MF, the University of Bologna, Italy ("Pallotti" fund to MF, "RFO" fund to ED, MF). EP in 2018 was a fellow of 
Fondazione Veronesi, Milano (Italy); MR in 2018 was a fellow of Fondazione Famiglia Parmiani, Bologna (Italy).

\section{Authors' contributions}

Conception and design: E. Dika, M. Ferracin

Development of methodology: E. Dika, E. Broseghini, E. Porcellini, M. Ferracin

Acquisition of data (provided animals, acquired and managed patients, provided

facilities, etc.): R. Roncarati, C. Misciali, M. Negrini, A. Patrizi, M. Lambertini, M. Riefolo

Analysis and interpretation of data (e.g., statistical analysis, biostatistics,

computational analysis): E. Broseghini, E. Porcellini, C. Bassi, G. Durante, P. Loher, I. Rigoutsos, E. Londin

Writing, review, and/or revision of the manuscript: E. Dika, E. Broseghini, E. Porcellini, M. Ferracin

Study supervision: E. Dika, M. Ferracin

\section{Acknowledgments}

Not applicable

\section{References}

1. Adler NR, Kelly JW, Haydon A, McLean CA, Mar VJ. Clinicopathological characteristics and prognosis of patients with multiple primary melanomas. Br J Dermatol 2018; 178: e44-e45.

2. Alonso SR, Tracey L, Ortiz P, Perez-Gomez B, Palacios J, Pollan M et al. A high-throughput study in melanoma identifies epithelial-mesenchymal transition as a major determinant of metastasis. Cancer Res 2007; 67: 3450-3460.

3. Anders S, Pyl PT, Huber W. HTSeq-a Python framework to work with high-throughput sequencing data. Bioinformatics 2015; 31: 166-169.

4. Bassoli S, Pellegrini C, Longo C, Di Nardo L, Farnetani F, Cesinaro AM et al. Clinical, dermoscopic, and confocal features of nevi and melanomas in a multiple primary melanoma patient with the MITF p.E318K homozygous mutation. Melanoma Res 2018; 28: 166-169.

5. Blackwood MA, Holmes R, Synnestvedt M, Young M, George C, Yang H et al. Multiple primary melanoma revisited. Cancer 2002; 94: 2248-2255.

6. Bruno W, Pastorino L, Ghiorzo P, Andreotti V, Martinuzzi C, Menin C et al. Multiple primary melanomas (MPMs) and criteria for genetic assessment: MultiMEL, a multicenter study of the Italian Melanoma Intergroup. J Am Acad Dermatol 2016; 74: 325-332. 
7. Cancer Genome Atlas N. Genomic Classification of Cutaneous Melanoma. Cell 2015; 161: 16811696.

8. Caramel J, Papadogeorgakis E, Hill L, Browne GJ, Richard G, Wierinckx A et al. A switch in the expression of embryonic EMT-inducers drives the development of malignant melanoma. Cancer Cell 2013; 24: 466-480.

9. Casula M, Paliogiannis P, Ayala F, De Giorgi V, Stanganelli I, Mandala M et al. Germline and somatic mutations in patients with multiple primary melanomas: a next generation sequencing study. BMC Cancer 2019; 19: 772.

10. Chawla G, Deosthale P, Childress S, Wu YC, Sokol NS. A let-7-to-miR-125 MicroRNA Switch Regulates Neuronal Integrity and Lifespan in Drosophila. PLoS Genet 2016; 12: e1006247.

11. Cloonan N, Wani S, Xu Q, Gu J, Lea K, Heater S et al. MicroRNAs and their isomiRs function cooperatively to target common biological pathways. Genome Biol 2011; 12: R126.

12. Colombino M, Sini M, Lissia A, De Giorgi V, Stanganelli I, Ayala F et al. Discrepant alterations in main candidate genes among multiple primary melanomas. J Transl Med 2014; 12: 117.

13. De Simone P, Valiante M, Silipo V. Familial melanoma and multiple primary melanoma. G Ital Dermatol Venereol 2017; 152: 262-265.

14. Di Lorenzo S, Fanale D, Corradino B, Calo V, Rinaldi G, Bazan V et al. Absence of germline CDKN2A mutation in Sicilian patients with familial malignant melanoma: Could it be a population-specific genetic signature? Cancer Biol Ther 2016; 17: 83-90.

15. Dika E, Patrizi A, Rossi C, Turchetti D, Miccoli S, Ferracin M et al. Clinical histopathological features and CDKN2A/CDK4/MITF mutational status of patients with multiple primary melanomas from Bologna: Italy is a fascinating but complex mosaic. G Ital Dermatol Venereol 2020.

16. Doubrovsky A, Menzies SW. Enhanced survival in patients with multiple primary melanoma. Arch Dermatol 2003; 139: 1013-1018.

17. El Sharouni MA, Witkamp AJ, Sigurdsson V, van Diest PJ. Comparison of Survival Between Patients With Single vs Multiple Primary Cutaneous Melanomas. JAMA Dermatol 2019.

18. Ferrone CR, Ben Porat L, Panageas KS, Berwick M, Halpern AC, Patel A et al. Clinicopathological features of and risk factors for multiple primary melanomas. JAMA 2005; 294: 1647-1654.

19. Gershenwald JE, Scolyer RA. Melanoma Staging: American Joint Committee on Cancer (AJCC) 8th Edition and Beyond. Ann Surg Oncol 2018; 25: 2105-2110.

20. Goto Y, Arigami T, Kitago M, Nguyen SL, Narita N, Ferrone S et al. Activation of Toll-like receptors 2, 3, and 4 on human melanoma cells induces inflammatory factors. Mol Cancer Ther 2008; 7: 36423653 .

21. Grossman D, Farnham JM, Hyngstrom J, Klapperich ME, Secrest AM, Empey S et al. Similar survival of patients with multiple versus single primary melanomas based on Utah Surveillance, Epidemiology, and End Results data (1973-2011). J Am Acad Dermatol 2018; 79: 238-244. 
22. Guarino M, Rubino B, Ballabio G. The role of epithelial-mesenchymal transition in cancer pathology. Pathology 2007; 39: 305-318.

23. Hao L, Ha JR, Kuzel P, Garcia E, Persad S. Cadherin switch from E- to N-cadherin in melanoma progression is regulated by the PI3K/PTEN pathway through Twist and Snail. Br J Dermatol 2012; 166: 1184-1197.

24. Hayward NK, Wilmott JS, Waddell N, Johansson PA, Field MA, Nones $\mathrm{K}$ et al. Whole-genome landscapes of major melanoma subtypes. Nature 2017; 545: 175-180.

25. Helgadottir $\mathrm{H}$, Tuominen $\mathrm{R}$, Olsson $\mathrm{H}$, Hansson J, Hoiom V. Cancer risks and survival in patients with multiple primary melanomas: Association with family history of melanoma and germline CDKN2A mutation status. J Am Acad Dermatol 2017; 77: 893-901.

26. Hsu MY, Meier FE, Nesbit M, Hsu JY, Van Belle P, Elder DE et al. E-cadherin expression in melanoma cells restores keratinocyte-mediated growth control and down-regulates expression of invasionrelated adhesion receptors. Am J Pathol 2000; 156: 1515-1525.

27. Hwa C, Price LS, Belitskaya-Levy I, Ma MW, Shapiro RL, Berman RS et al. Single versus multiple primary melanomas: old questions and new answers. Cancer 2012; 118: 4184-4192.

28. Ivanova NB, Dimos JT, Schaniel C, Hackney JA, Moore KA, Lemischka IR. A stem cell molecular signature. Science 2002; 298: 601-604.

29. Johnson TM, Hamilton T, Lowe L. Multiple primary melanomas. J Am Acad Dermatol 1998; 39: 422427.

30. Kalluri R, Neilson EG. Epithelial-mesenchymal transition and its implications for fibrosis. J Clin Invest 2003; 112: 1776-1784.

31. Krebsbach PH, Villa-Diaz LG. The Role of Integrin alpha6 (CD49f) in Stem Cells: More than a Conserved Biomarker. Stem Cells Dev 2017; 26: 1090-1099.

32. Kreizenbeck GM, Berger AJ, Subtil A, Rimm DL, Gould Rothberg BE. Prognostic significance of cadherin-based adhesion molecules in cutaneous malignant melanoma. Cancer Epidemiol Biomarkers Prev 2008; 17: 949-958.

33. Le MT, Xie H, Zhou B, Chia PH, Rizk P, Um M et al. MicroRNA-125b promotes neuronal differentiation in human cells by repressing multiple targets. Mol Cell Biol 2009; 29: 5290-5305.

34. Lee RC, Feinbaum RL, Ambros V. The C. elegans heterochronic gene lin-4 encodes small RNAs with antisense complementarity to lin-14. Cell 1993; 75: 843-854.

35. Loher P, Rigoutsos I. Interactive exploration of RNA22 microRNA target predictions. Bioinformatics 2012; 28: 3322-3323.

36. Loher P, Londin ER, Rigoutsos I. IsomiR expression profiles in human lymphoblastoid cell lines exhibit population and gender dependencies. Oncotarget 2014; 5: 8790-8802.

37. Love MI, Huber W, Anders S. Moderated estimation of fold change and dispersion for RNA-seq data with DESeq2. Genome Biol 2014; 15: 550. 
38. Lynch HT, Krush AJ. Heredity and malignant melanoma: implications for early cancer detection. Can Med Assoc J 1968; 99: 17-21.

39. Mao B, Zhang Z, Wang G. BTG2: a rising star of tumor suppressors (review). Int J Oncol 2015; 46: 459-464.

40. Maubec E, Chaudru V, Mohamdi H, Blondel C, Margaritte-Jeannin P, Forget S et al. Familial melanoma: clinical factors associated with germline CDKN2A mutations according to the number of patients affected by melanoma in a family. J Am Acad Dermatol 2012; 67: 1257-1264.

41. Menzies S, Barry R, Ormond P. Multiple primary melanoma: a single centre retrospective review. Melanoma Res 2017; 27: 638-640.

42. Mort RL, Jackson IJ, Patton EE. The melanocyte lineage in development and disease. Development 2015; 142: 620-632.

43. Murali R, Goumas C, Kricker A, From L, Busam KJ, Begg CB et al. Clinicopathologic features of incident and subsequent tumors in patients with multiple primary cutaneous melanomas. Ann Surg Oncol 2012; 19: 1024-1033.

44. Potrony M, Badenas C, Aguilera P, Puig-Butille JA, Carrera C, Malvehy J et al. Update in genetic susceptibility in melanoma. Ann Transl Med 2015; 3: 210.

45. Ramalho-Santos M, Yoon S, Matsuzaki Y, Mulligan RC, Melton DA. "Stemness": transcriptional profiling of embryonic and adult stem cells. Science 2002; 298: 597-600.

46. Riefolo M, Porcellini E, Dika E, Broseghini E, Ferracin M. Interplay between small and long non-coding RNAs in cutaneous melanoma: a complex jigsaw puzzle with missing pieces. Mol Oncol 2019; 13: 74-98.

47. Rouault JP, Falette N, Guehenneux F, Guillot C, Rimokh R, Wang Q et al. Identification of BTG2, an antiproliferative p53-dependent component of the DNA damage cellular response pathway. Nat Genet 1996; 14: 482-486.

48. Savoia P, Osella-Abate S, Deboli T, Marenco F, Stroppiana E, Novelli M et al. Clinical and prognostic reports from 270 patients with multiple primary melanomas: a 34-year single-institution study. J Eur Acad Dermatol Venereol 2012; 26: 882-888.

49. Silye R, Karayiannakis AJ, Syrigos KN, Poole S, van Noorden S, Batchelor W et al. E-cadherin/catenin complex in benign and malignant melanocytic lesions. J Pathol 1998; 186: 350-355.

50. Takazawa Y, Kiniwa Y, Ogawa E, Uchiyama A, Ashida A, Uhara H et al. Toll-like receptor 4 signaling promotes the migration of human melanoma cells. Tohoku J Exp Med 2014; 234: 57-65.

51. Tang A, Eller MS, Hara M, Yaar M, Hirohashi S, Gilchrest BA. E-cadherin is the major mediator of human melanocyte adhesion to keratinocytes in vitro. J Cell Sci 1994; 107 (Pt 4): 983-992.

52. Telonis AG, Magee R, Loher P, Chervoneva I, Londin E, Rigoutsos I. Knowledge about the presence or absence of miRNA isoforms (isomiRs) can successfully discriminate amongst 32 TCGA cancer types. Nucleic Acids Res 2017; 45: 2973-2985. 
53. Tse JC, Kalluri R. Mechanisms of metastasis: epithelial-to-mesenchymal transition and contribution of tumor microenvironment. J Cell Biochem 2007; 101: 816-829.

54. Utjes D, Lyth J, Lapins J, Eriksson H. Reduced disease-specific survival following a diagnosis of multiple primary cutaneous malignant melanomas-a nationwide, population-based study. Int $\mathrm{J}$ Cancer 2017; 141: 2243-2252.

55. Wagener N, Bulkescher J, Macher-Goeppinger S, Karapanagiotou-Schenkel I, Hatiboglu G, AbdelRahim $\mathrm{M}$ et al. Endogenous BTG2 expression stimulates migration of bladder cancer cells and correlates with poor clinical prognosis for bladder cancer patients. Br J Cancer 2013; 108: 973-982.

56. Wei CY, Wang L, Zhu MX, Deng XY, Wang DH, Zhang SM et al. TRIM44 activates the AKT/mTOR signal pathway to induce melanoma progression by stabilizing TLR4. J Exp Clin Cancer Res 2019; 38: 137.

57. Wei S, Hao C, Li X, Zhao H, Chen J, Zhou Q. Effects of BTG2 on proliferation inhibition and antiinvasion in human lung cancer cells. Tumour Biol 2012; 33: 1223-1230.

58. Yang $\mathrm{CH}$, Yue J, Pfeffer SR, Handorf CR, Pfeffer LM. MicroRNA miR-21 regulates the metastatic behavior of B16 melanoma cells. J Biol Chem 2011; 286: 39172-39178.

59. Zhang L, Huang H, Wu K, Wang M, Wu B. Impact of BTG2 expression on proliferation and invasion of gastric cancer cells in vitro. Mol Biol Rep 2010; 37: 2579-2586.

\section{Figures}




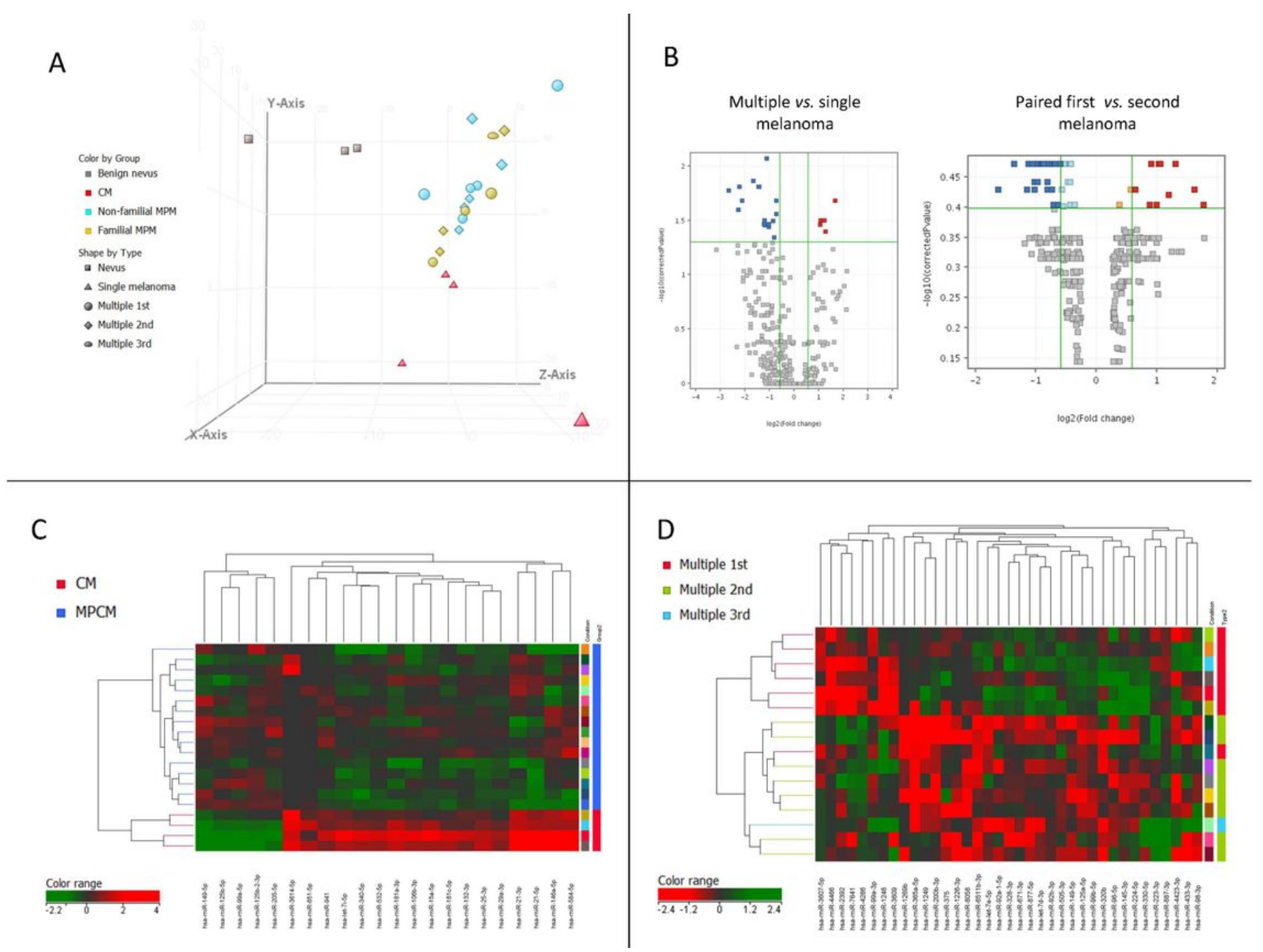

\section{Figure 1}

Principal Component Analysis (PCA) of samples analyzed by smallRNA sequencing. A) Familial (yellow) and non-familial (cyan) multiple primary melanomas display a similar microRNA profile, which is different from single cutaneous melanoma (red) and benign nevi (grey). B) Heatmap of multiple and single melanoma based on the expression of 22 differentially expressed miRNAs (moderated t-test, adjusted $\mathrm{p}<0.05)$. Red and green color represent the increased or reduced expression across samples. $\mathrm{C}$ ) Heatmap of the first and second melanomas from the same patient based on the expression of 37 differentially expressed miRNAs (paired t-test, $p<0.05$ ). Red and green color represent the increased or reduced expression across samples. D) Volcano plot showing the differentially expressed miRNAs at the selected $p$-value and fold change combinations. 

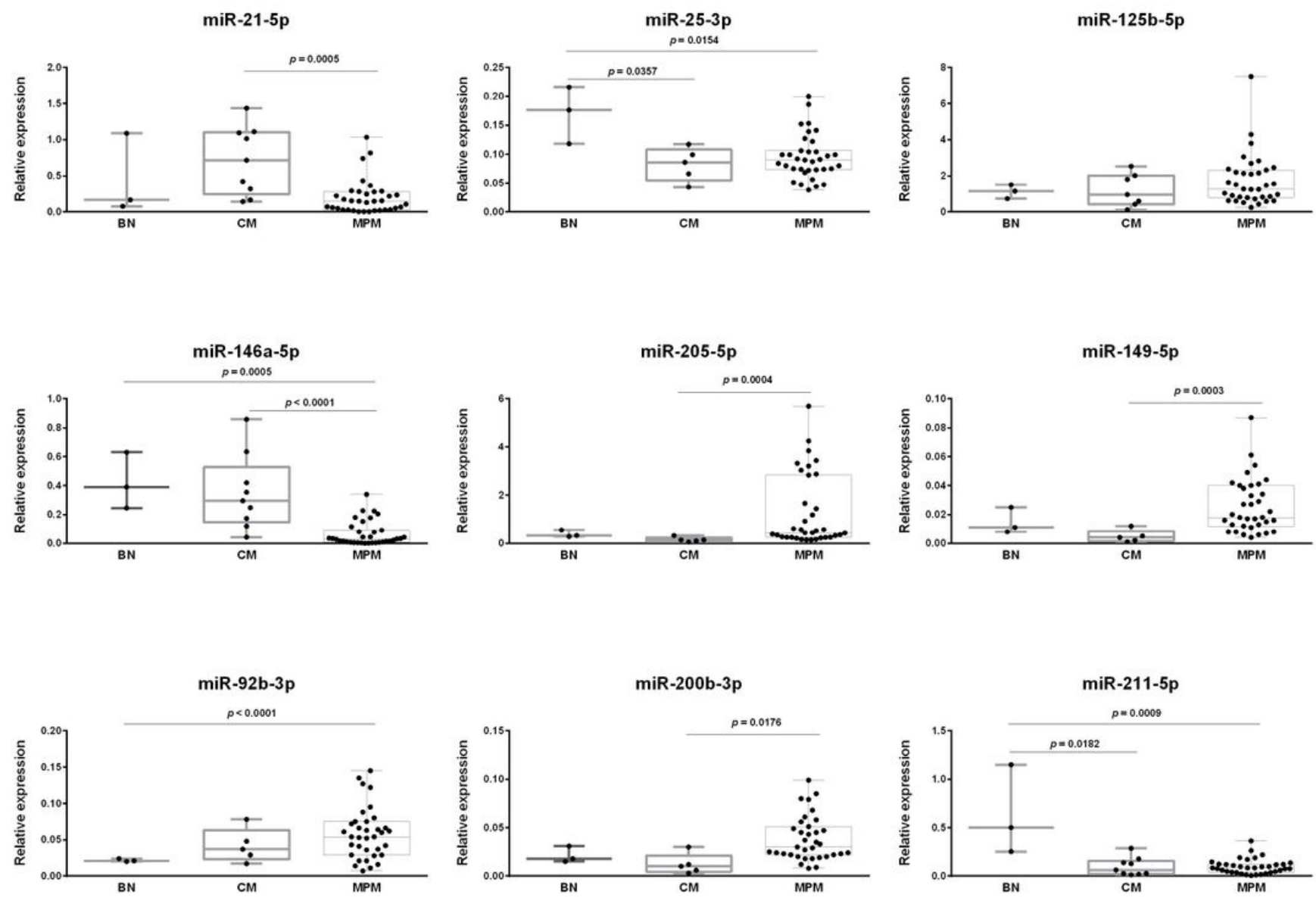

Figure 2

Differential microRNA expression in benign nevi (BN), cutaneous melanoma (CM) and in multiple primary melanoma (MPM). Dot plot representation of 9 microRNAs differentially expressed in single and multiple primary melanomas $(P<0.05)$. MPM shows higher expression levels of miR-205-5p, miR-200b-3p, miR149-5p compared to $\mathrm{CM}$, and higher expression of miR-92b-3p compared to BN. MPM downregulates miR-21-5p compared to CM and miR-146a-5p compared to CM and BN. BN upregulates miR-25-3p and miR-211-5p compared to CM and MPM. Each miRNA was tested in triplicate by quantitative RT-PCR. Relative miRNA expression was normalized on invariant miR-16-5p. 
miR-149-5p

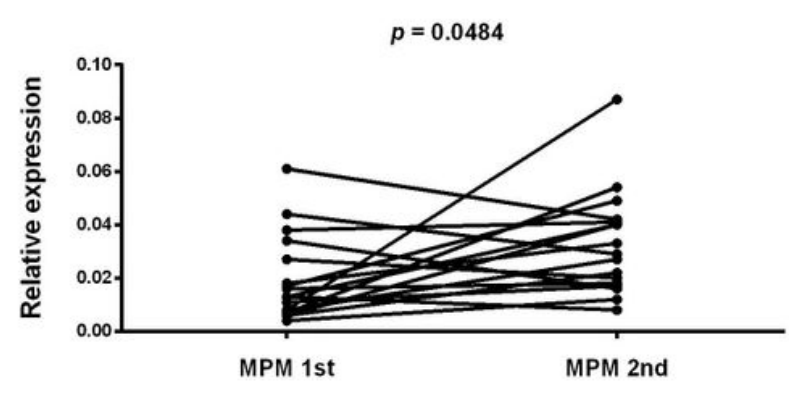

miR-205-5p

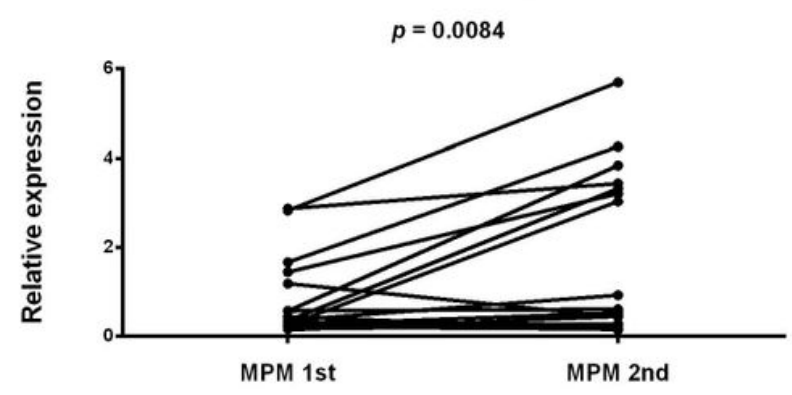

miR-92b-3p

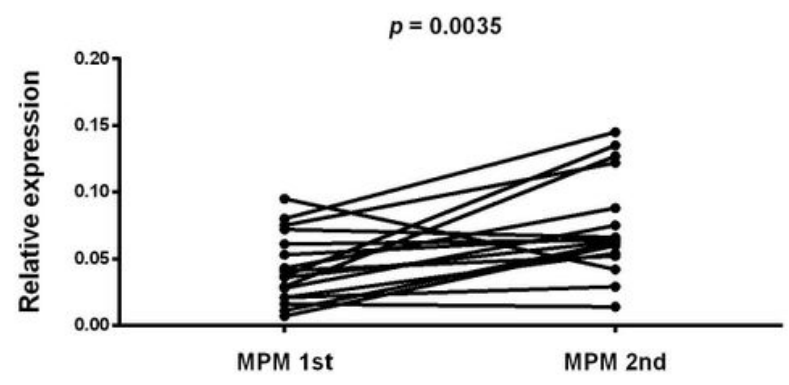

miR-200b-3p

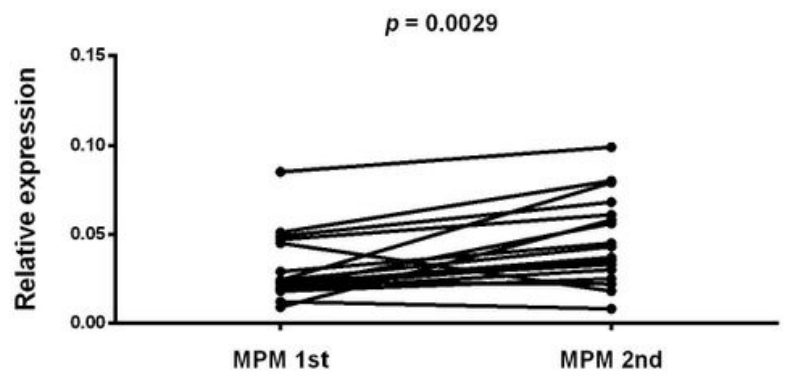

\section{Figure 3}

Differential microRNA expression in 1st vs. 2nd melanoma from the same patient. Before-after plot showing the paired expression of 4 selected microRNAs in 17 multiple primary melanoma (MPM) patients. miR-92b-3p, miR-205-5p, miR-200b-5p and miR-149-5p are significantly downregulated in the 1 st melanoma compared to the 2nd melanoma. Each miRNA was tested in triplicate by quantitative RT-PCR. Relative miRNA expression was normalized on invariant miR-16-5p. Paired P-value is reported. 


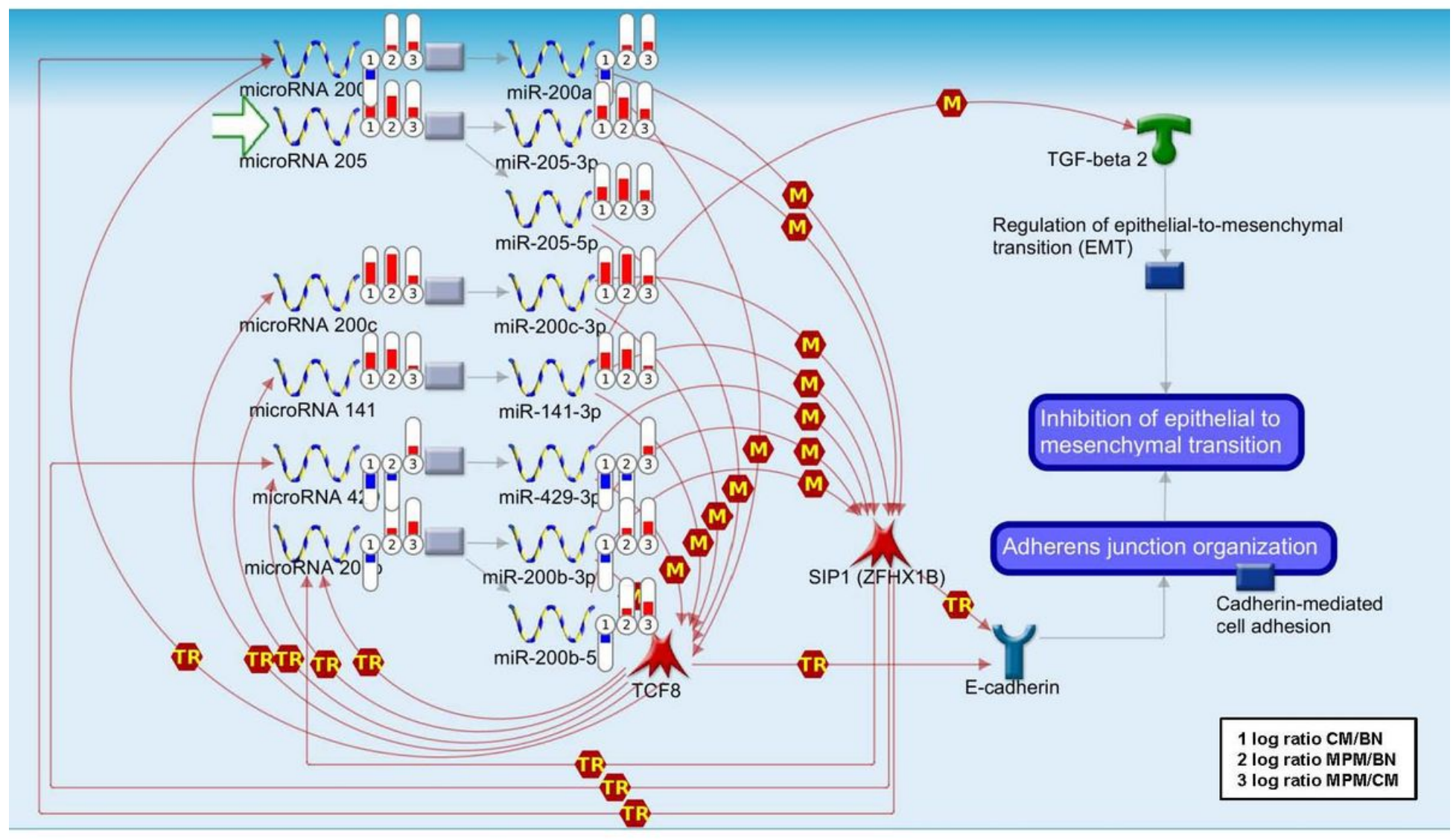

\section{Figure 4}

MetaCore pathway analysis showing the involvement of differently expressed miRNAs in epithelialmesenchymal transition (EMT). EMT pathway representation with regulating miRNAs. Log ratio of miRNA expression level in CM/BN (1), MPM/BN (2), MPM/CM (3) is visualized on the maps as a thermometerlike figure. Upward thermometers have red color and indicate upregulated signals and downward (blue) ones indicate downregulated expression level of specific microRNAs. MPM showed higher expression levels of microRNAs involved in the inhibition of epithelial-mesenchymal transition (EMT), including miR205-5p and miR-200b-3p. (BN, benign nevi; CM, cutaneous melanoma; MPM multiple primary melanoma). 
A

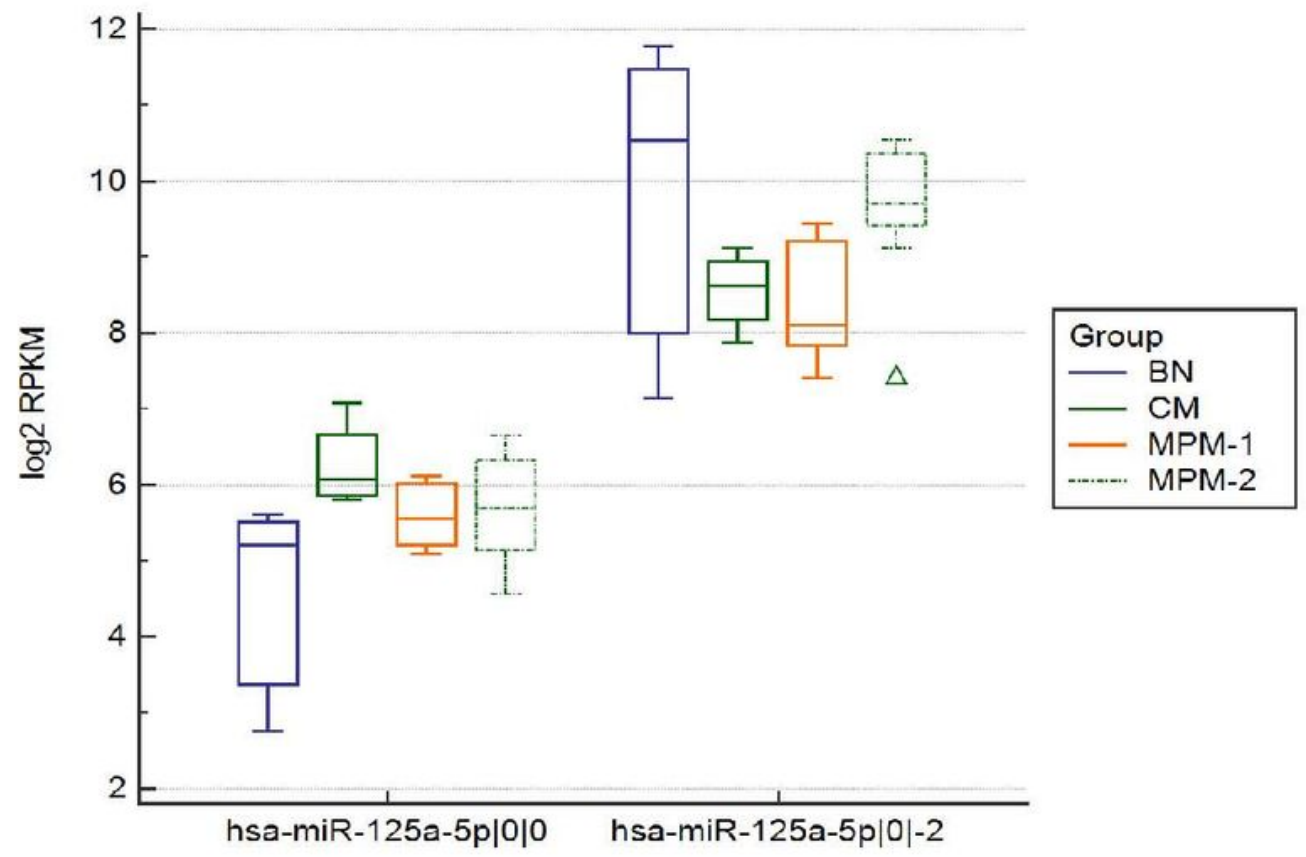

B

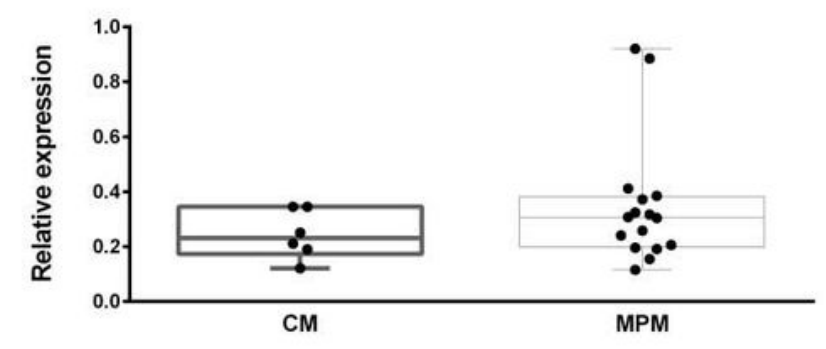

miR-125a-5p (short+long)

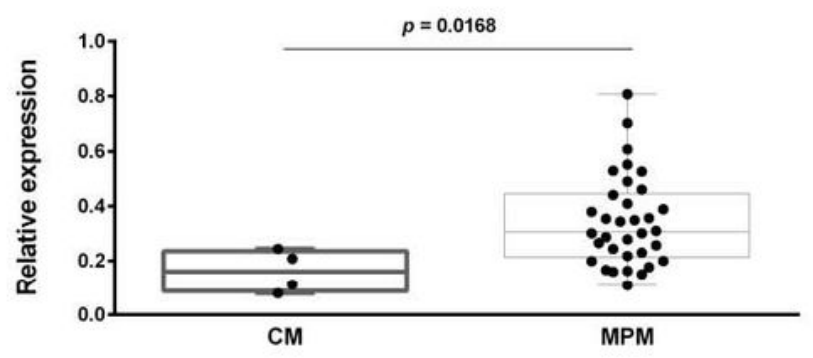

C

miR-125a-5p (long)

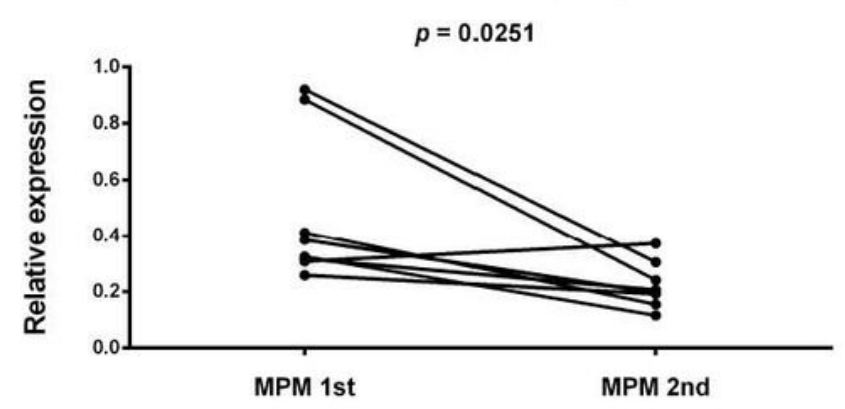

miR-125a-5p (short+long)

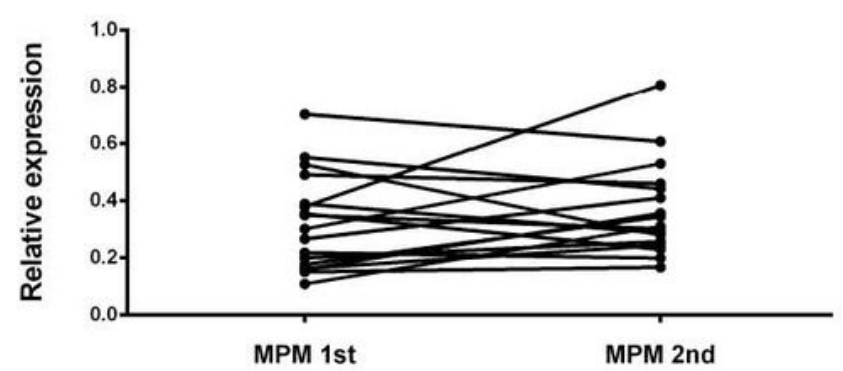

\section{Figure 5}

Comparison of canonical and isomiR miR-125a-5p expression trends in benign nevi (BN), cutaneous melanoma (CM) and multiple primary melanoma (MPM). A. Box-plot graph of canonical miR-125a-5p (hsa-miR-125a-5p|0|0) showing a lower expression level and opposite expression trend in BN, CM and MPM if compared to its shorter isomiR (hsa-miR-125a-5p|0|-2). B. Dot plot representation of canonical miR-125a-5p expression assessed with miRCURY LNA assay, which is specific for the canonical form, 
and overall expression of all miR-125a-5p isoforms, detected using miSCRIPT assay. Results show that the combined expression of miR-125a-5p isoforms of levels is higher in MPM compared to CM. C. Beforeafter plot of canonical miR-125a-5p expression (miRCURY LNA assay) and all miR-125a-5p isoforms (miSCRIPT assay) shows an opposite trend in 1st and 2nd melanoma from the same patient.

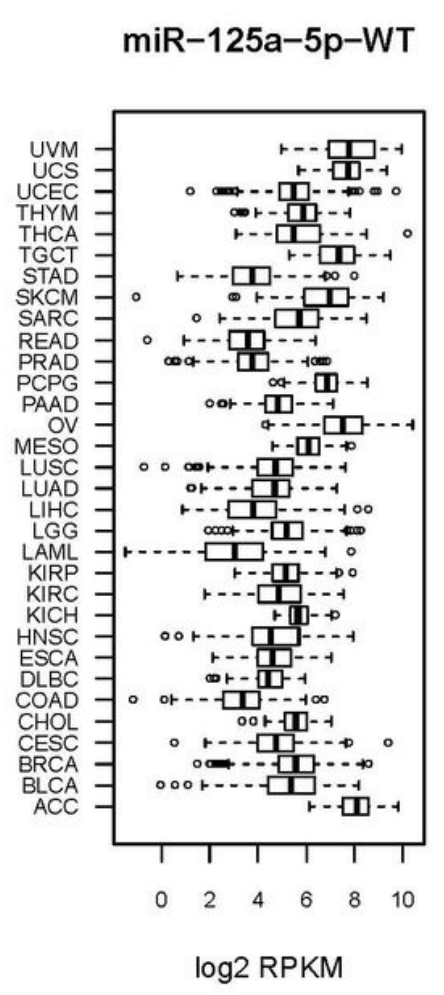

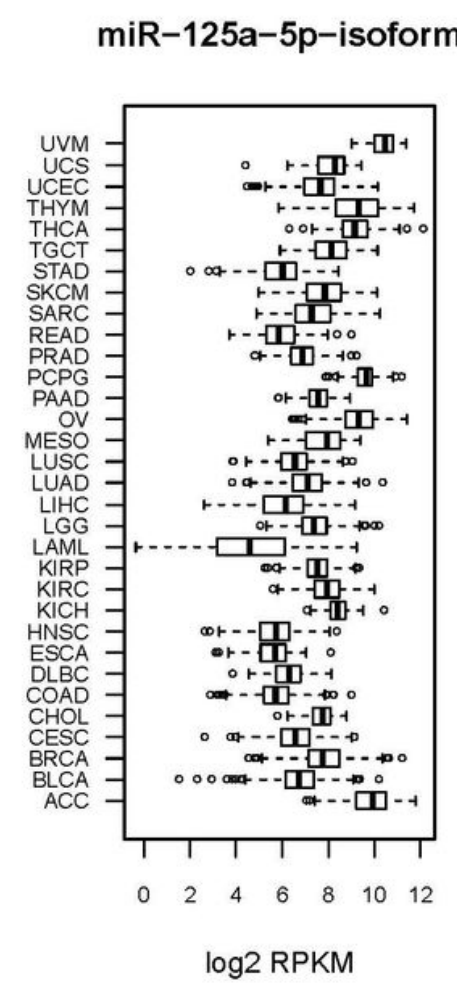

$\log 2$ RPKM

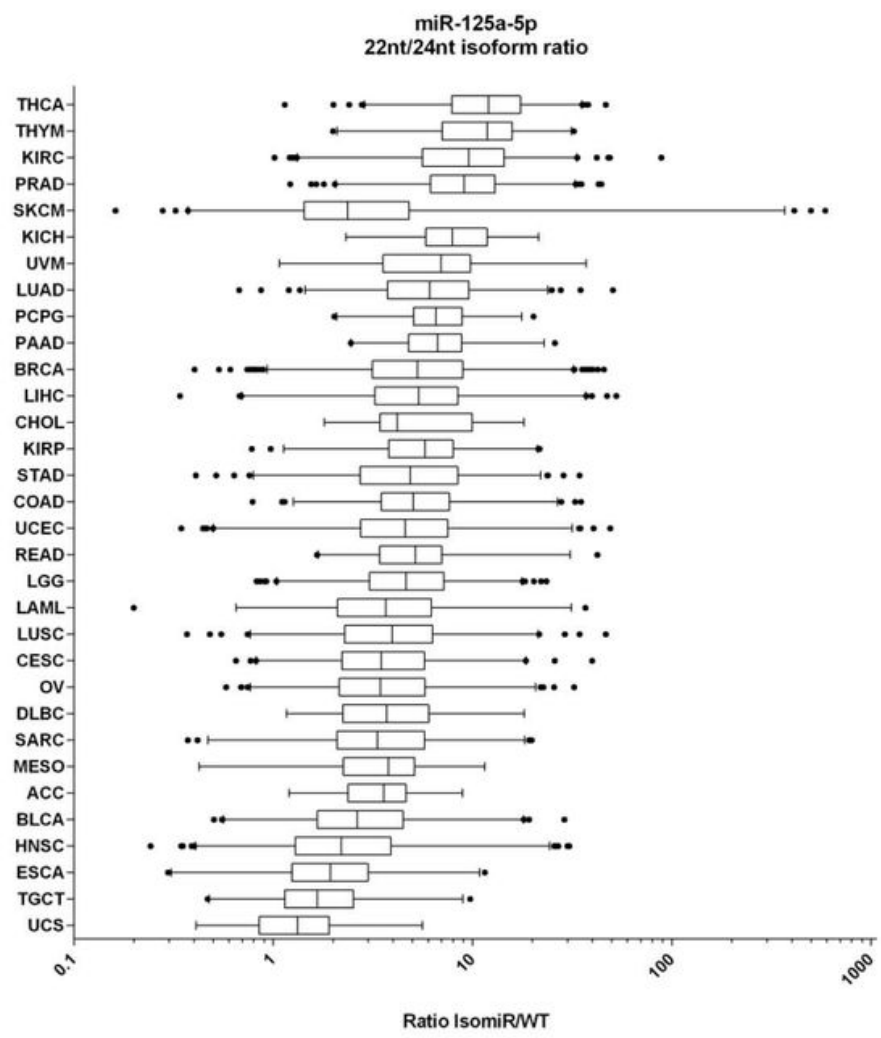

Ratio IsomiRWT

\section{Figure 6}

Canonical (wild-type) and isomiR analysis of miR-125a-5p across 32 TCGA tumor types. miR-125a isomiR is most expressed expressed in many cancer types. Box plots of canonical (WT) and shorter isoform of miR-125a-5p show variable expression levels, represented here as log2 RPKM data, across 32 different cancer type. miR-125a-5p isoform is most abundant in many cancer types, and shows a specifically high canonical/isoform ratio in melanoma (SKCM) group. TCGA abbreviations: ACC, adrenocortical carcinoma; BLCA, bladder urothelial carcinoma; BRCA, breast invasive carcinoma; CESC, cervical squamous cell carcinoma and endocervical adenocarcinoma; $\mathrm{CHOL}$, cholangiocarcinoma; $\mathrm{COAD}$, colon adenocarcinoma; DLBC, lymphoid neoplasm diffuse large B-cell lymphom; ESCA, esophageal carcinoma; HNSC, head and neck squamous cell carcinoma; KICH, kidney chromophobe; KIRC, kidney renal clear cell carcinoma; KIRP, kidney renal papillary cell carcinoma; LAML, acute myeloid leukemia; LGG, brain lower grade glioma; LIHC, liver hepatocellular carcinoma; LUAD, lung adenocarcinoma; LUSC, lung squamous cell carcinoma; MESO, mesothelioma; OV, ovarian serous cystadenocarcinoma; PAAD, pancreatic adenocarcinoma; PCPG, pheochromocytoma and paraganglioma; PRAD, prostate adenocarcinoma; READ, rectum adenocarcinoma; SARC, sarcoma; SKCM, skin cutaneous melanoma; STAD, stomach adenocarcinoma; TGCT, testicular germ cell tumors; THCA, thyroid carcinoma; THYM, 
thymoma; UCEC, uterine corpus endometrial carcinoma; UCS, uterine carcinosarcoma; UVM, uveal melanoma.

\section{Supplementary Files}

This is a list of supplementary files associated with this preprint. Click to download.

- Additionalfile3.pdf

- Additionalfile3.pdf

- Additionalfile3.pdf

- Additionalfile2.pdf

- Additionalfile2.pdf

- Additionalfile2.pdf 